\title{
PHILelergite
}

\section{EL PRESENTE DE INDICATIVO ESPAÑOL Y LA PERSPECTIVA COGNITIVA: SUBJETIVIZACIÓN Y DOMINIOS DE CONTROL*}

\author{
DANA KRATOCHVÍLOVÁ \\ Univerzita Karlova
}

\begin{abstract}
RESUMEN: Este artículo analiza el presente de indicativo español (el paradigma HABLO) desde la perspectiva de los dominios de control y el grado de subjetivización que presentan sus distintos usos. Trabajando con el modelo evolutivo dinámico de Langacker (1991), llegamos a la conclusión de que es posible establecer el valor central de dicho paradigma basándonos en el concepto de dominio de control perceptivo.

PALABRAS CLAVE: lingüística cognitiva, presente de indicativo, subjetivización, dominio de control epistémico, dominio de control efectivo, modelo evolutivo dinámico
\end{abstract}

The present indicative in Spanish and the cognitive perspective: subjectification and dominions of control

\begin{abstract}
The following article analyzes the present indicative in Spanish (the HABLO paradigm) from the perspective of dominions of control and the degree of subjectification that can be attributed to its different uses. Working with Langacker's dynamic evolutionary model (1991), we conclude that it is possible to establish the central value of this paradigm on the basis of the concept of dominion of perceptive control.

KEYWORDS: cognitive linguistics, present indicative, subjectification, dominion of epistemic control, dominion of effective control, dynamic evolutionary model
\end{abstract}

\section{INTRODUCCIÓN}

Es un hecho bien conocido que el paradigma HABLO (o el tradicionalmente llamado 'presente de indicativo') tiene una gran escala de posibles usos. Puede representar procesos de extensión temporal muy variable (desde unos cuantos segundos hasta varios milenios) y sustituir a los paradigmas HABLARÉ (Mañana \{estoy estaré\} en Madrid), HABLÓ (En 1492 Colón \{descubre descubrió\} América) e incluso a las formas del imperativo (iSe lo dices ahora mismo! iDíselo ahora mismo!). En las oraciones condicionales, HABLO se refiere a hechos hipotéticos (Si tengo tiempo, te llamaré). Dada su polifuncionalidad, los distintos empleos de este paradigma no se prestan fácilmente a una clasificación unánime, lo que en la tradición lingüística ha llevado a considerar dicho paradigma como no marcado, neutral o como el candidato menos problemático en distintos contextos.

En trabajos anteriores donde estudiábamos la oposición entre el empleo del subjuntivo y el indicativo, planteábamos nuestras dudas acerca de la posibilidad de atribuirle a una forma 
verbal un estatuto permanente de miembro marcado / no marcado de la oposición (cf. Kratochvílová, 2013, 2018 en prensa). Continuando con dicha línea de investigación, en este artículo presentamos un análisis de los distintos usos del paradigma HABLO basado en la lingüística cognitiva, concretamente en el modelo evolutivo dinámico (dynamic evolutionary model) (Langacker, 1991) y en los conceptos de subjetivización (subjectification) (Langacker, 1990, 1999, 2006) y de dominios de control efectivo y epistémico (dominion of effective control, dominion of epistemic control) (Langacker, 2009; Vesterinen, 2014).

Partiendo de las propuestas de Vesterinen (2014) y Mejías-Bikandi (2009), defendemos que las nociones de dominio son esenciales en la descripción de los valores de las formas verbales y proponemos una tercera esfera: el dominio de control perceptivo. Estudiaremos los distintos usos del paradigma HABLO desde la perspectiva cognitivista y estableceremos su valor general: la correspondencia parcial o total del proceso verbal con el dominio de control perceptivo del hablante. Mediante la relación entre el dominio de control perceptivo y la incorporación implícita del hablante en el proceso verbalmente representado, comprobaremos cómo los conceptos de dominio están estrechamente ligados con la noción de subjetivización y cómo el grado de subjetivización es un elemento clave en la distinción de los diferentes usos del paradigma HABLO.

El artículo se organiza de la siguiente manera. En el segundo apartado presentamos brevemente el modelo evolutivo dinámico de Langacker, que más tarde servirá como base para nuestras representaciones gráficas de los usos del paradigma HABLO. Los apartados tercero y cuarto introducen la noción de los dominios de control y la de la subjetivización. En el tercer apartado estudiamos, además, la noción de dominio de control perceptivo y su relación con los dominios de control epistémico y efectivo. El quinto apartado se centra en los métodos de investigación utilizados en este trabajo, mientras que el sexto analiza los usos del paradigma HABLO, haciendo énfasis en su relación con el dominio de control perceptivo y el grado de subjetivización que presentan.

\section{El MODELO EVOLUTIVO DINÁMICO}

Según Langacker (1991), la realidad y el paso del tiempo pueden representarse de la siguiente manera:

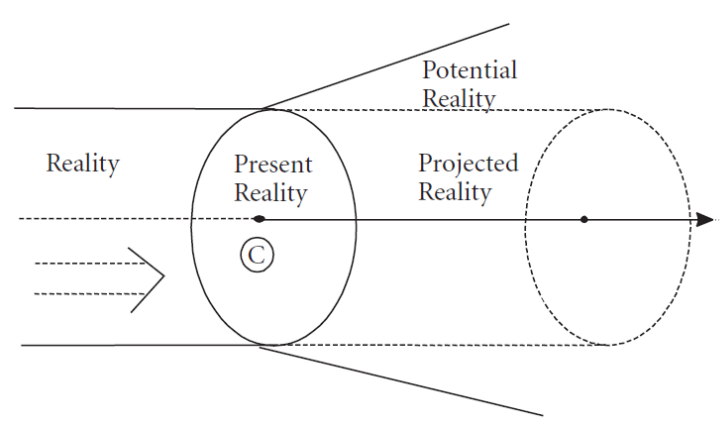

Figura 1. Dynamic evolutionary model (Langacker, 1991)

Este modelo muestra cómo un conceptualizador (C) concibe los eventos en el paso del tiempo. La realidad (reality) está formada por los eventos que el conceptualizador da por reales, o 
sucedidos. La realidad inmediata (present reality) coincide con el momento del habla y funciona como el punto de anclaje desde el cual el hablante presenta todos los sucesos. En el marco de la irrealidad se encuentran la realidad proyectada (projected reality), que se refiere a los sucesos que aún no han tenido lugar, pero que el conceptualizador concibe como relativamente ciertos; y la realidad potencial (potential reality), que está formada por los eventos cuya realización futura no está excluida por parte del conceptualizador.

Aplicando este modelo al sistema verbal español, en términos muy generales se puede constatar que la realidad se expresaría mediante el uso de los tiempos del indicativo tradicionalmente concebidos como pretéritos. La realidad inmediata correspondería al uso del presente de indicativo. El futuro simple y el futuro compuesto de indicativo se referirían a la realidad proyectada, mientras que el subjuntivo, el imperativo y el condicional representarían la realidad potencial. No obstante, esta visión es muy simplificada, como demuestran Achard (2000) o Castañeda Castro (2004), quienes presentan un análisis más elaborado del modelo langackeriano aplicado al verbo español, donde cuestionan algunos de los valores tradicionalmente atribuidos a los paradigmas verbales. Aún más allá van las observaciones de Ruiz Campillo (2014, 65), quien constata que "cualquier 'tiempo' [verbal] parece poder usarse para cualquier tiempo (cronológico o lingüístico)” y propone una representación figurativa del sistema verbal que no opera con nociones como la del "presente de indicativo' o el 'imperfecto de subjuntivo', sino con la distinción básica entre los modos declarativos (los tradicionales 'indicativo' y 'condicional') y el modo no-declarativo (el 'subjuntivo'). A estos se los divide no desde la perspectiva del presente o del pasado, sino desde el punto de vista del espacio en el que se encuentra el hablante (espacio actual - aquí) o en el que se sitúa mentalmente (espacio inactual - allí).

Dichos estudios demuestran la problemática y polifacética naturaleza de los tiempos y modos verbales españoles y, atendiendo a la amplia gama de sus posibles usos, ponen en duda las tradicionales etiquetas de 'presente' y 'pretérito', 'indicativo' e 'imperativo', etc. Basándonos en dicha línea de investigación, sostenemos que las funciones de los distintos paradigmas verbales no se orientan, principalmente, según las nociones de tiempo, sino según los dominios de control del hablante, los cuales muchas veces se corresponden con la organización temporal tradicional. No obstante, dicha correspondencia no es absoluta.

\section{DOMINIOS DE CONTROL EPISTÉMICO, EFECTIVO Y PERCEPTIVO}

Presentando la oposición entre el control efectivo y el control epistémico, Langacker (2009, 153) constata que " $[t]$ hese aspects of linguistic structure reflect not only our constant effort to acquire knowledge about the world, but also our efforts to change it - besides just learning what happens, we try to influence what happens". Según Langacker, los dominios de control efectivo y de control epistémico son elementos clave a la hora de representar verbalmente tanto los procesos como los objetos. Hablando de los verbos y de los procesos, el control efectivo del sujeto lo representan el imperativo o los verbos modales en su interpretación deóntica. El control epistémico se asocia con el conocimiento del hablante, o sea, con el modo indicativo y con la ausencia de verbos modales en su interpretación epistémica.

Siguiendo las propuestas de Langacker, Vesterinen (2014) aplica el concepto de dominio al uso del subjuntivo en español y en portugués. Este autor elabora la propuesta de Maldonado (1995), según la cual el subjuntivo en los contextos epistémicos indica que un proceso se halla 
fuera del dominio epistémico del hablante, y también relaciona la noción de dominio con el control efectivo (las capacidades del sujeto para causar, cambiar o influir en un proceso). Vesterinen $(2014,613)$ propone así un valor central del modo subjuntivo, que sería el de designar un suceso localizado fuera del dominio del conceptualizador: "it designates an event that is located outside the conceptualizer's dominion. The linguistic expression triggering mood choice indicates the relevant dominion [control epistémico o control efectivo].”

Dichos análisis demuestran la importancia de la noción de control sobre un evento y su relación con la selección de una forma verbal concreta. No obstante, en ninguno de esos análisis se plantea explícitamente la cuestión de si los dominios de control efectivo y de control epistémico son los únicos que influyen de alguna manera en la representación verbal de procesos o entidades. En este estudio sostenemos que hay un tercer dominio que se debe considerar: el dominio de control perceptivo. Dicho dominio correspondería a nuestra capacidad de ver, sentir, oír, oler o entrar en algún tipo de contacto perceptivo con un objeto o un proceso. Todos los procesos sometidos al control perceptivo entran también en el dominio de control epistémico del hablante, ya que, observando o entrando en contacto perceptivo con una entidad o un proceso, reconocemos su existencia. Sin embargo, esta correspondencia no se da en el sentido opuesto porque hay objetos o eventos que consideramos reales sin que tengamos que entrar en interacción perceptiva con ellos.

La división de los dominios perceptivo y epistémico se corresponde perfectamente con la situación en el mundo real. Si nos quedamos quietos y con la mirada fija, los objetos que están frente a nosotros serán el contrapunto de los procesos que entran en el dominio del control perceptivo. Estas cosas las vemos y, por lo tanto, las concebimos como reales. Imaginemos, por ejemplo, que estamos observando un paisaje. Delante de nosotros se extiende un prado que termina en un bosque: la parte del prado y del bosque que podemos captar con la vista, el canto de los pájaros que podemos oír, el olor de la hierba, etc. corresponden al dominio de control perceptivo. No obstante, lo que efectivamente vemos, oímos, olemos o sentimos en un momento determinado es solo una pequeña parte de las cosas que conocemos y consideramos reales. Volviendo a la escena del prado y del bosque, es posible que sepamos, por ejemplo, que hay un pueblo detrás del bosque y que hay un camino que lleva al prado (incluso puede ser que hayamos llegado al prado por ese mismo camino). No podemos ver el pueblo (los árboles del bosque nos lo impiden) ni el camino (está detrás de nosotros), pero sabemos de su existencia. El pueblo y el camino forman, por lo tanto, parte de nuestro control epistémico.

La oposición entre el control epistémico y el control perceptivo se refleja en la lengua. En el sistema nominal correspondería a la diferencia entre el uso de los demostrativos este, ese o aquel (objetos que entran en la esfera de nuestro control perceptivo) y el artículo definido (esfera del control epistémico). En el sistema verbal, el control perceptivo correspondería al uso de las formas del presente de indicativo. En cambio, las formas del pretérito y del futuro de indicativo se relacionarían con el control epistémico.

A diferencia de Vesterinen $(2014,598)$, para quien el dominio de control efectivo tiene una extensión menor que el dominio de control epistémico y entra en él, consideramos que esta esfera está completamente apartada del control perceptivo y del epistémico. El control efectivo se relaciona con nuestra capacidad de cambiar el mundo y causar o provocar procesos nuevos, transformando así las entidades que nos rodean. Estos cambios no pueden estar sometidos a nuestro control epistémico o perceptivo, ya que todavía no han tenido lugar y no podemos tener la certeza de si sucederán efectivamente o no. En el modelo evolutivo de Langacker, los 
dominios de control epistémico y perceptivo formarían parte de la realidad conocida (inmediata y proyectada), mientras que el dominio de control efectivo se hallaría en la realidad potencial. En lo que se refiere al reflejo de dicho dominio en el sistema verbal, "[i]n the case of clauses, the grounding elements employed for purposes of effective control are root modals and the basic imperative constructions" (Langacker, 2009, 167),

Concluimos, pues, que en líneas generales los dominios de control se reflejan en el sistema verbal español de la siguiente manera: el control epistémico sería el paradigma HABLÉ, el control perceptivo corresponde a HABLO, y el control efectivo a iHABLA!. Los demás paradigmas verbales se relacionan con nociones más complejas cuya función no se puede resumir en términos de coincidencia con uno de los dominios.

\section{SUBJETIVIZACIÓN}

La subjetivización, en el sentido langackeriano del término, se puede identificar con la inclusión implícita de la base (ground), o sea, la situación en la que se pronuncia un enunciado y que incluye al hablante, en la estructuración conceptual (construal). Cuanto más saliente sea la base en la estructuración conceptual, más objetivamente construida estará y menor será el grado de subjetivización. Véase, por ejemplo, la diferencia entre las siguientes frases: Yo, ahora, estoy en Madrid Estoy en Madrid. Si bien el contenido semántico es más o menos el mismo en las dos, la diferencia consiste en el grado de objetividad con que se presentan el hablante y el momento en que se produce la enunciación, que a su vez forman la base. Usando el pronombre yo y el adverbio ahora, tanto el momento de la enunciación como el conceptualizador aparecen explícitamente en la estructuración conceptual, por lo que se los presenta como totalmente objetivos. El segundo ejemplo ofrece un mayor grado de subjetivización, ya que tanto la perspectiva del hablante como la localización temporal quedan fuera de escena, no se representan objetivamente y solo se deducen de la forma del verbo estar. Resulta, pues, que los morfemas de tiempo y modo (junto con los verbos modales) funcionan como los llamados elementos de anclaje (grounding elements), que establecen una relación entre una entidad o un proceso y la base (cf. Langacker, 1990, 1999, 2006). ${ }^{1}$

En este estudio proponemos que los distintos usos del paradigma HABLO se pueden clasificar según el grado de subjetivización que presentan. Sostenemos también que dicha noción está estrechamente ligada a la de dominio de control perceptivo.

\section{ENFOQUE DEL ANÁLISIS Y REPRESENTACIÓN GRÁFICA}

Siguiendo las líneas de investigación introducidas en los apartados anteriores, presentamos un análisis que se centra en el paradigma HABLO, situando sus distintos valores temporales y modales en el modelo langackeriano. Trabajaremos con una versión modificada del modelo evolutivo dinámico, introduciendo en él la visualización del hablante y de la situación comunicativa en la que se formula un enunciado (el dominio de control perceptivo).

Para distinguir con claridad entre el hablante en la situación comunicativa y el proceso, representaremos la situación comunicativa y el control perceptivo del hablante como un cubo geométrico. La extensión espacial del cubo corresponde a la extensión temporal de la situación comunicativa, mientras que las líneas que dan forma al cubo (o, dicho de otra manera, que dan forma concreta a la situación comunicativa en la que se encuentra el conceptualizador) 
representan el control perceptivo del hablante sobre los sucesos que conceptualiza en dicha situación comunicativa.

La extensión temporal de la situación comunicativa es más larga que el momento de la enunciación. Por tanto, la situación comunicativa se representa tridimensionalmente y el momento de la enunciación se concibe bidimensionalmente como un plano que atraviesa el cubo y coincide con la realidad inmediata: ${ }^{2}$

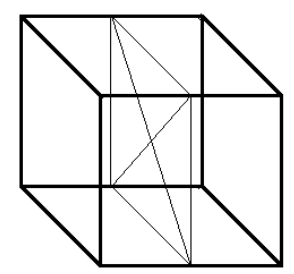

Figura 2. Dominio de control perceptivo y momento de la enunciación

$\mathrm{Al}$ representar verbalmente un proceso con el paradigma HABLO, el cubo contendrá el cilindro langackeriano que corresponde a la extensión temporal del proceso y su coincidencia o no coincidencia con el momento del habla y con el dominio de control perceptivo del conceptualizador. El plano del momento de la enunciación separará entonces el pasado (o la realidad conocida) del futuro (o la realidad proyectada).

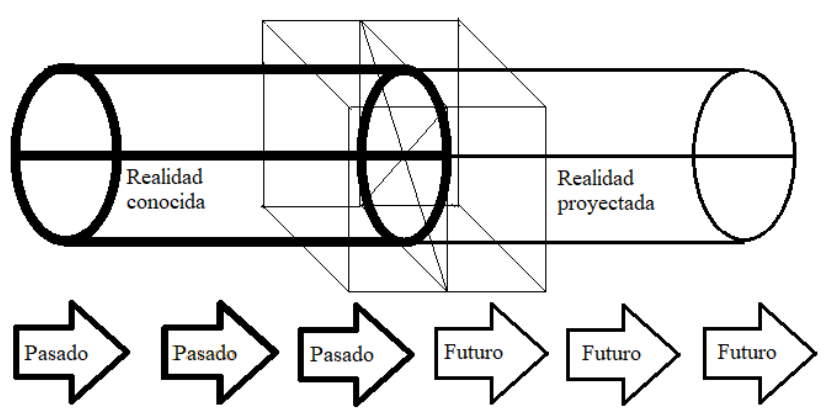

Figura 3. Representación gráfica de un proceso verbal

La diferencia en el ancho de las líneas sirve para marcar el grado de inclusión de las nociones que se representan en la estructuración conceptual. El hablante y la situación comunicativa, a menos que se vean mencionados explícitamente (por ejemplo, mediante el pronombre personal), siempre se construyen como implícitos (la línea más fina que forma el cubo y el plano del momento de la enunciación). El cilindro representa el proceso verbal, el cual se construye objetivamente y está en el centro de atención. En el caso del paradigma HABLO, la parte del proceso que se relaciona con el pasado (o la realidad conocida) es más saliente, ya que comprende los sucesos que ya han sido dados por seguros. Si bien varios usos del paradigma HABLO se extienden también más allá del momento de la enunciación, esta extensión siempre se da por supuesta porque el conceptualizador no conoce el futuro. Por lo 
tanto, la dirección de la extensión temporal que va hacia la realidad proyectada resulta menos saliente en la representación verbal del proceso. A diferencia de Langacker, no usamos la línea intermitente para representar la realidad proyectada. Esta nos servirá más adelante para la representación del desplazamiento mental del control perceptivo del hablante que, sin embargo, no está incluido en la semántica del verbo.

\section{VALORES DEL PARADIGMA HABLO}

A continuación, emplearemos la representación gráfica que presentamos en el apartado anterior para el análisis de los distintos valores del paradigma HABLO. Analizaremos dicha representación desde el punto de vista del grado de subjetivización y veremos cómo el dominio de control perceptivo del hablante forma parte de ella, demostrando de este modo su función esencial en la semántica de los procesos expresados mediante este paradigma. Todos los ejemplos que usamos provienen del corpus CORPES XXI (ver. o.83), creado por la RAE y accesible en www.rae.es. Las búsquedas realizadas a lo largo de todo el corpus tienen como objetivo aportar ejemplos claros y auténticos procedentes de distintos países de habla hispana. Debajo de cada cita proporcionamos la información sobre su autor, la obra de la que ha sido tomada, el país, el año de publicación y el género al que pertenece.

\subsection{Simultaneidad absoluta}

La simultaneidad absoluta de un proceso con el momento de la enunciación se obtiene con los usos performativos de verbos como pedir, declarar, prometer, etc.:

(1) Ahora firma, sólo te pido eso.

Lillo, Daniela. Carita de emperaora. Chile, 2001, ficción (teatro).

Dada la coincidencia de la extensión temporal del proceso expresado por el paradigma HABLO con el momento de la enunciación, su representación gráfica es bidimensional:

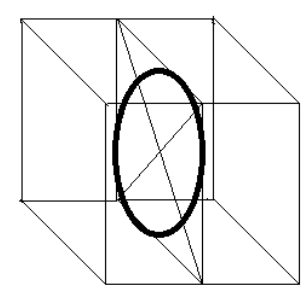

Figura 4. Simultaneidad absoluta

La representación figurativa refleja el control absoluto que tiene el hablante sobre el proceso que representa verbalmente. Puesto que el conceptualizador y el proceso coinciden en tiempo y espacio, el conceptualizador se percibe como saliente y construido objetivamente. La base completa y el punto de perspectiva (vantage point) también son salientes e inseparables del proceso verbal. Por lo tanto, el grado de subjetivización es mínimo, como lo es la extensión temporal del proceso, que comprende por lo general solo unos cuantos segundos. 


\subsection{Simultaneidad perceptiva}

La simultaneidad perceptiva comprende los usos de verbos de percepción física como ver, oír, oler, sentir, etc. cuando estos se refieren a una percepción continua. Se relaciona asimismo con el empleo de los verbos que expresan acontecimientos susceptibles de ser percibidos por los sentidos (pueden ser observados, oídos, etc.):

(2) Minutos después entra la enfermera. Presiento que se acerca la media noche por el silencio que impera.

Quezada, Roberto. Los potros del recuerdo. Guatemala, 2001, ficción (novela).

Incluye también el empleo de los verbos de pensamiento o de reacción emotiva cuando estos se refieren a sucesos momentáneos, generalmente causados directamente por un suceso previo:

(3) Me detengo en mis reflexiones y pienso inmediatamente en la categoría que tenía el gran estilista y "catedrático" torrelaveguense, Chiqui Linares.

Cabello, Modesto. Los bolos. Recuerdos y reflexiones. España, 2001, no ficción (ensayo).

Algunos de estos usos del paradigma HABLO corresponderían al presente progresivo, que se caracteriza por su posible sustitución por estar + gerundio (cf. RAE, 2009, 1710). No obstante, dicho criterio se basa en una característica aspectual del proceso (su progresividad), sin tomar en cuenta otros posibles valores de este uso del paradigma HABLO. 3

De acuerdo con la línea de argumentación que seguimos en este trabajo, consideramos que el aspecto clave aquí lo constituye el control perceptivo continuo por parte del hablante sobre el proceso en cuestión. Por su naturaleza, dicho control no puede durar infinitamente y se traduce en una extensión temporal relativamente corta del proceso (segundos, minutos $\mathrm{u}$ horas, generalmente). El control continuo se refleja en la siguiente representación gráfica:

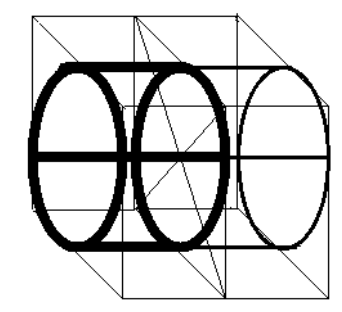

Figura 5. Simultaneidad perceptiva

El cilindro que representa el proceso verbal atraviesa todo el cubo de la situación comunicativa, sobrepasando el momento de la enunciación, lo cual corresponde a la extensión temporal general de la simultaneidad perceptiva. Al representar verbalmente un proceso de percepción sensorial o mental mediante el paradigma HABLO, el hablante suele aludir a procesos que empezaron antes del momento de la enunciación y se espera que continúen en el futuro. Los límites del proceso coinciden con los dos lados del cubo, es decir, con el dominio de control perceptivo del hablante, lo cual está condicionado por la participación activa de los sentidos o de la mente. Esto hace que el hablante resulte saliente e inseparable del proceso. No obstante, 
el nivel de subjetivización es ligeramente mayor que en el caso de la simultaneidad absoluta porque los límites del proceso sobrepasan el momento de la enunciación, o, lo que es lo mismo, el punto de orientación temporal, que, por tanto, resulta menos saliente y más subjetivo.

\subsection{Simultaneidad perceptiva virtual}

Los verbos de percepción física y los que indican acciones perceptibles por los sentidos pueden pasan a referirse a procesos que no están bajo el control continuo del hablante y solo se presentan como tales, lo cual les permite adquirir una extensión temporal más larga (horas, días, semanas, meses). Dado este control virtual sobre el proceso, hablamos de simultaneidad perceptiva virtual. Hay una diferencia intuitiva en el significado de los verbos está, hablan y veo que aparecen en los siguientes ejemplos y en la expectativa de la extensión temporal del proceso que denominan. Mientras que los ejemplos (4), (5) y (6) expresan la simultaneidad perceptiva, los ejemplos (7), (8) y (9) se relacionan con la simultaneidad perceptiva virtual:

Simultaneidad perceptiva

(4) Román está en la puerta de la tienda apoyado en el quicio, mirando hacia la lejanía invisible.

Cano Pavón, José Manuel. El hombre sitiado. España, 2003, ficción (teatro).

(5) Mientras caminan por la calle, hablan de tonterías.

Escudos, Jacinta. El desencanto. El Salvador, 2001, ficción (relato).

(6) A través de la ventana, veo a mi vecina arriba de una escalera encalando el muro.

Rimsky M., Cynthia. Poste restante. Chile, 2001, ficción (novela).

Simultaneidad perceptiva virtual

(7) Mi coche está en el taller.

Osorio, Amaranta. Teatro. Piezas breves. Alumnos RESAD. Curso 2003/2004. México, 2004, ficción (teatro).

(8) Hace mucho que algunos hablan de dejar Maiokati y nosotras queremos partir también. Biggs, Jorge. En torno a la casa de Madame Lorraine. Chile, 2001, ficción (novela).

(9) Antes me despertaba y lo primero que veía eran los olivos. Ahora desde la ventana veo los perros revolviendo la basura.

Fernández Barreyro, Fabián. Un mar de mieles. Argentina, 2007, ficción (teatro).

Dicha diferencia consiste en la naturaleza del control perceptivo que tiene el conceptualizador sobre el proceso indicado por el verbo. En el caso de la simultaneidad perceptiva virtual, los procesos expresados por el paradigma HABLO se entienden como repetidos a lo largo de un período de tiempo (los verbos hablar y ver en las pautas algunos hablan de dejar Maiokati y veo los perros revolviendo la basura) o como procesos continuos que, no obstante, deberían terminar en un futuro no muy lejano (el verbo estar en la pauta mi coche está en el taller). El aspecto clave de la simultaneidad perceptiva virtual es su incompatibilidad con las 
características inmutables de las cosas que no están sujetas al control del hablante. Los ejemplos usados suponen su participación en alguno de estos valores:

\begin{tabular}{|l|l|}
\hline \multicolumn{1}{|c|}{ Proceso } & \multicolumn{1}{c|}{ Posible participación perceptiva } \\
\hline \multirow{2}{*}{ (A) Mi coche está en el taller. } & $\begin{array}{l}\text { Lo he visto en el taller. (Lo he llevado allí yo } \\
\text { mismo o he ido al taller para verlo). }\end{array}$ \\
\cline { 2 - 2 } & $\begin{array}{l}\text { Me han dicho que lo llevaron allí. (He oído } \\
\text { esta información). }\end{array}$ \\
\hline $\begin{array}{l}\text { (B) Hace mucho que algunos hablan de } \\
\text { dejar Maiokati. }\end{array}$ & Los he oído personalmente. \\
\cline { 2 - 2 } & $\begin{array}{l}\text { Lo he leído en un periódico u oído en la radio } \\
\text { o en la televisión. }\end{array}$ \\
\hline $\begin{array}{l}\text { (C) Ahora desde la ventana veo los perros } \\
\text { revolviendo la basura. }\end{array}$ & Los he visto con mis propios ojos. \\
\hline
\end{tabular}

Tabla 1. Control perceptivo en la simultaneidad perceptiva virtual

Como podemos observar, la participación real de los sentidos del hablante generalmente no coincide con la propia situación comunicativa y se sitúa en el pasado. Sin embargo, el uso del paradigma HABLO (en vez de algunos de los paradigmas principalmente atribuidos a los tiempos 'pasados' o 'pretéritos') hace que la participación activa del hablante y su control perceptivo se perciban como efectivos a lo largo de toda la extensión temporal del proceso. Este hecho se refleja en la representación figurativa de la simultaneidad perceptiva virtual:

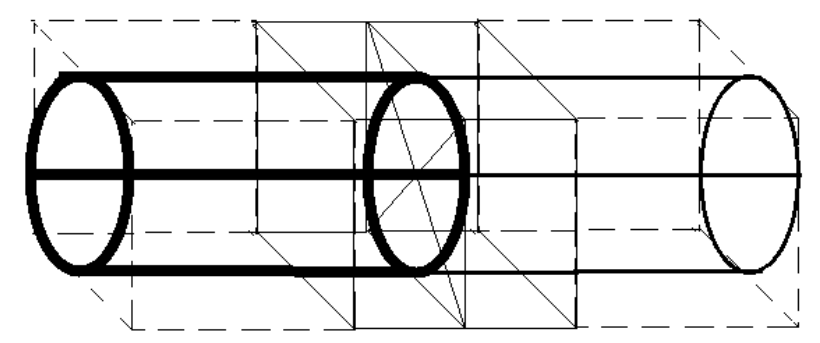

Figura 6. Simultaneidad perceptiva virtual

Una vez más, el cilindro representa el proceso verbalmente expresado y su extensión temporal, la raya más ancha representa la parte del proceso que corresponde a la realidad conocida, y la raya más fina es la parte del proceso que comprende la realidad proyectada (la supuesta extensión temporal del proceso más allá del momento de la enunciación). El cubo central representa la situación comunicativa y el control perceptivo del hablante, mientras que las rayas intermitentes que crean los demás cubos corresponden al desplazamiento mental del control perceptivo del hablante y limitan la extensión del cilindro (proceso verbal), que no puede ir más allá del control perceptivo proyectado del conceptualizador.

Dada la necesaria participación del conceptualizador, este no puede proyectar la duración de los procesos en un futuro que escapa a su control. En el caso de los verbos de percepción física o mental, la continuación del proceso sin la participación del hablante queda completamente excluida. Si el hablante del ejemplo (9) deja de mirar por la ventana por las 
mañanas o se muda a otra casa, no podrá ver los perros y el proceso verbalmente representado por la forma verbal veo terminará. En el caso de los verbos que se refieren a procesos perceptibles por los sentidos o por la mente, el cese del control del hablante supondría su dislocación del campo de la realidad proyectada a la esfera de la realidad potencial, en la cual no podrían expresarse verbalmente mediante el simple paradigma HABLO. Fijémonos en el efecto que producen los siguientes enunciados:

Llevo meses sin ir o llamar al taller, \{pero supongo que mi coche está allí pero mi coche debería estar allí ??pero mi coche está allí\}.

Me he ido de Maiokati \{y supongo que la gente alli sigue hablando de partir también y la gente allí seguirá hablando de partir también ??y la gente allí sigue hablando de partir también\}.

La ausencia del control perceptivo virtual del hablante se puede marcar mediante el verbo suponer, el verbo modal deber o el paradigma HABLARÉ en su uso de 'futuro epistémico'. Su empleo hace que dichas pautas resulten naturales y no se perciba contradicción lógica en ellas. El simple uso del paradigma HABLO, por el contrario, implica la siguiente contradicción: 'he dejado de ejercer el control sobre algo, pero sigo teniéndolo bajo mi control.' Dicha contradicción desaparece solo gracias a la presuposición de otro modo en el cual el hablante pueda ejercer el control perceptivo virtual sobre el proceso (por ejemplo, informarse a través de otra persona, de los periódicos o situarse mentalmente en el taller o en Maiokati).

Vemos, pues, que el conceptualizador tiene que estar presente, por lo menos virtualmente, en todas las fases del proceso. No obstante, es esta virtualidad lo que lo hace menos saliente y menos construido objetivamente que en los casos anteriores. También la base y la perspectiva desde la que se observa el proceso se construyen de manera más subjetiva, ya que el proceso los sobrepasa notablemente.

Como observamos en la tabla 1, la simultaneidad perceptiva virtual admite, además, una clara interpretación en términos de evidencialidad, lo cual demuestra la estrecha relación de esta categoría con las nociones de anclaje y subjetividad (cf. Langacker, 2017). Si identificamos el control perceptivo del hablante con la evidencia sensorial, se corrobora indirectamente también la propuesta de Bermúdez (2006, 2011, 2016) de que la evidencialidad se puede encontrar en el sistema verbal del español.

\subsection{Simultaneidad extensiva basada en la experiencia del hablante}

En un gran número de usos, el paradigma HABLO se refiere a procesos cuya extensión temporal comprende meses o años y la propia situación comunicativa se corresponde solo con una pequeña parte de dicha extensión.

(10) Es buena persona, afectuoso y tiene cierta rigidez en los valores que defiende. Olivera, Lucila. Clarín. Argentina, 2001, no ficción (prensa).

El inicio de estos procesos se corresponde con el nacimiento (en el caso de seres vivos) o con la creación (en el caso de cosas) del sujeto, con su madurez o con el momento en que ha tenido lugar algún cambio con consecuencias permanentes. Estos procesos ya no pueden ser 
controlados constantemente por el hablante y no entran del todo en la esfera de su control perceptivo (real o virtual), solo llegando a atravesarla. El control por parte del conceptualizador se ejerce en términos de la propia experiencia personal o de sus conocimientos. Podría representarse explícitamente mediante verbos como sé que, noto que, me parece que... De no ser expresadas verbalmente, las nociones que apuntan a la perspectiva del conceptualizador y a su modo de ver las cosas quedan implícitas y construidas subjetivamente. La ausencia de control perceptivo continuo hace que el hablante resulte también más subjetivo, que su papel se vea reducido al aporte de la orientación temporal y de sus experiencias o su punto de vista, así como que la atención se centre más en el mismo proceso.

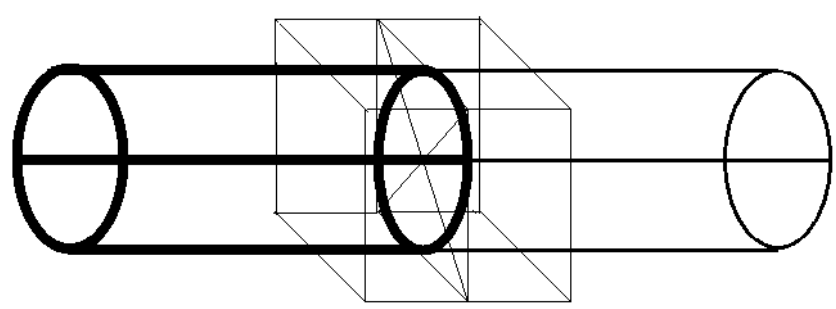

Figura 7. Simultaneidad extensiva basada en la experiencia del hablante

\subsection{Simultaneidad atemporal}

En su extensión máxima, la simultaneidad representada por el paradigma HABLO se refiere a procesos que no están limitados temporalmente. Su origen no está en la experiencia del conceptualizador, sino en el conocimiento enciclopédico o en la experiencia común:

(11) La rotación del eje polar de la Tierra dura 25.800 años y su descubrimiento, hace 22 siglos, se lo debemos a Hiparco, el mayor astrónomo de la antigüedad.

Ospina de la Roche, Arturo. El legado maya. Los aportes de un pueblo sorprendente a las matemáticas y la astronomía. Colombia, 2001, no ficción (ensayo).

(12) Sabido es que el Doberman posee una lealtad extraordinariamente feroz, impulsiva, francamente entregada a la agresividad que le viene de sus complicadísimos cruzamientos: batallan en su sangre los Levingstones, los Dogos, los Rotweilers, y otros.

De la Vega, Ricardo. Los hombres ya no invitan a cenar. Paraguay, 2001, ficción (relato).

Los sujetos de dichos procesos pueden ser entidades únicas (la Tierra, el Sol) que están sometidas a un estudio científico que no está vinculado a la experiencia individual que el conceptualizador pueda tener de ellas:

Simultaneidad perceptiva

(13) El aire huele a sal. El sol me quema los huesos.

Di Masso, Gerardo. El mar lejos. Argentina, 2002, ficción (novela). 
Simultaneidad extensiva

(14) Habla Julián Sánchez, 43 años, socorrista de la Cruz Roja y profesor de primeros auxilios. Uno de esos tipos experimentados - lleva 20 años de oficio- a los que ya nada sorprende. O casi nada: "El sol me da cada vez más miedo", asegura.

Rego, Paco. «Asustados por el cambio climático». Elmundo.es. Magazine. España, 2005, no ficción (prensa).

Simultaneidad atemporal

(15) Pero en realidad, el Sol es sólo un flujo de energía en forma de ondas electromagnéticas de diferentes frecuencias (luz visible, infrarroja y ultravioleta).

Conejo-Mir Sánchez, Julián. Aprende a conocer tu piel. Para tenerla sana y guapa. España, 2009, no ficción (ensayo).

Asimismo, el sujeto puede referirse a representantes de toda una clase de objetos o individuos. En este caso se representan sus características generales:

Simultaneidad perceptiva

(16) El perro entra al casco de la vieja hacienda por un agujero en el muro de adobe. Aridjis, Homero. La zona del silencio. México, 2001, ficción (novela).

Simultaneidad extensiva

(17) -El perro es mío - Tásai lo acaricia.

Aridjis, Homero. La zona del silencio. México, 2001, ficción (novela).

Simultaneidad atemporal

(18) El perro es uno de los peores masticadores de la naturaleza (ansioso e impaciente). "Los canarios deben salir de sus jaulas para pasear". Los Tiempos. Bolivia, 2001, no ficción (prensa).

El carácter objetivo de la información que se proporciona sobre el sujeto hace que la extensión temporal del paradigma HABLO extienda notablemente no tan solo los límites de la situación comunicativa, sino también los límites de la experiencia y el conocimiento individuales del conceptualizador. En la representación gráfica, este hecho lo representa la parte gris del cilindro:

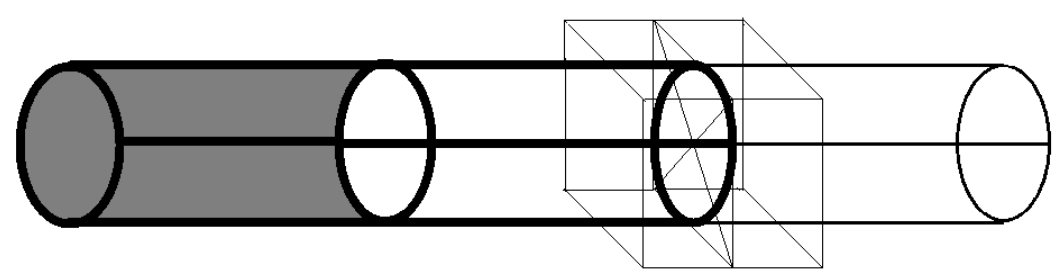

Figura 8. Simultaneidad atemporal 
Hasta ahora, todos los procesos representados verbalmente por el paradigma HABLO se encontraban bajo el alcance de la experiencia individual del conceptualizador y su capacidad de proyectar una evolución esperable en el futuro. Las partes transparentes del cilindro representan los límites de dicho alcance. Estas partes están 'iluminadas' por la conciencia del conceptualizador. En el caso de la simultaneidad atemporal, el hablante también representa verbalmente una parte del proceso que está fuera del alcance de sus propios conocimientos. La ausencia de experiencia la refleja la parte no transparente del cilindro, que se encuentra 'a oscuras' y supera los límites de la conciencia del hablante. Este funciona aquí como simple aportador de información y el papel de su control perceptivo queda reducido al mínimo. Alcanzamos un altísimo nivel de subjetivización que, a su vez, corresponde a la representación extremadamente objetiva del proceso, donde este se encuentra en el centro de atención y el hablante queda fuera de la escena, siendo su única incorporación la representación verbal (la formulación del enunciado), que supone el aporte del anclaje temporal.

\subsection{Anterioridad actualizada}

Como 'presente histórico', el paradigma HABLO puede sustituir al paradigma HABLÉ y referirse a eventos pasados:

(19) Y para colmo ese proceso comienza en el 94, el año en que estalló el escándalo por Europa del AMI, que fue lo mismo a escala universal. [...] Clinton invitó a sus amigos a Miami y de pronto se aparece con lo mismo: el TLC de México extendido a toda América Latina.

Elizalde, Rosa Miriam. "El levantamiento del bloqueo jamás significaría un peligro para la cultura cubana”. La Jiribilla. Cuba, 2001, no ficción (prensa).

Veiga (1987) y Briz (2004) opinan que dichos usos del paradigma HABLO no implican la dislocación de un evento pasado al presente, sino el desplazamiento del punto de referencia del momento actual al pasado (el hablante se sitúa mentalmente en el pasado). En este artículo, en cambio, sostenemos que el desplazamiento se produce en términos de control perceptivo. Si bien el momento mismo de la enunciación no se puede trasladar, el dominio de control perceptivo sí puede disociarse de él y desplazarse virtualmente al pasado. Desde este punto de vista, dichos usos se asimilan a la simultaneidad perceptiva virtual, ya que un proceso que no puede ser efectivamente controlado por los sentidos es presentado como si lo fuera. La diferencia consiste en que, en el caso de la simultaneidad perceptiva virtual, una parte de la extensión temporal del proceso se corresponde con el momento del habla, mientras que en el caso de la anterioridad actualizada la extensión temporal del proceso no incluye la actualidad y el momento de la enunciación:
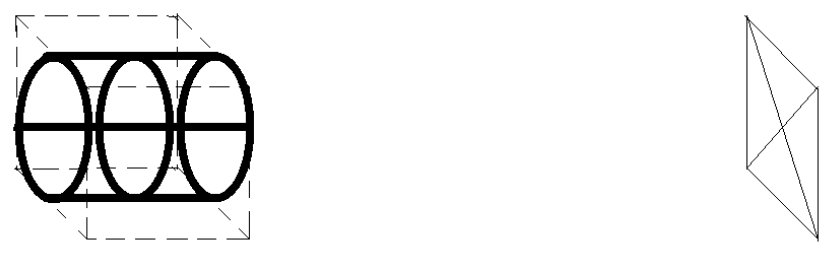

Figura 9. Anterioridad actualizada 
El cilindro que representa el proceso verbal se encuentra completamente fuera de la situación comunicativa. No obstante, el control perceptivo del hablante se mantiene en su proyección virtual. Dicha virtualidad y también el hecho de que el proceso esté desvinculado del momento de la enunciación, que es un elemento fundamental de la base, hacen que esta se construya como mayormente subjetiva y la atención se centre por completo en el proceso. Así se explican también los efectos estilísticos tradicionalmente asociados a este tipo de usos del paradigma HABLO, como despertar el interés del interlocutor por el proceso e involucrarlo en él presentando el proceso con más intensidad.

El desplazamiento virtual del dominio de control perceptivo del hablante y la coincidencia del proceso con este representa, además, la diferencia entre los usos de los paradigmas HABLO y HABLÉ en la referencia a sucesos pasados:
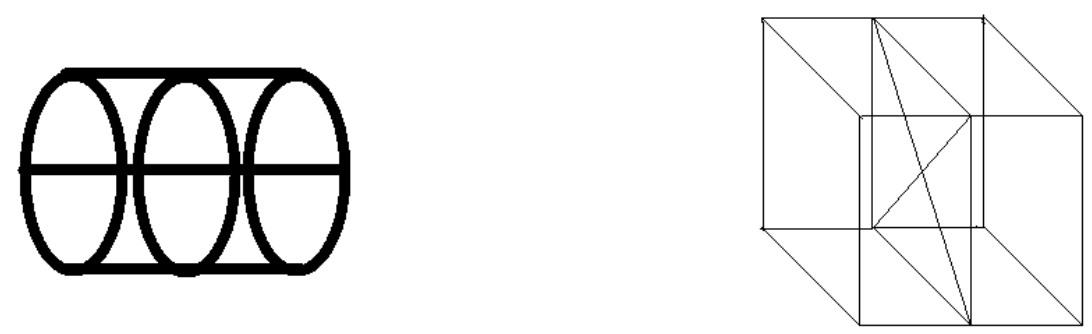

Figura 10. Anterioridad expresada mediante el paradigma HABLÉ

Desde el punto de vista de la subjetivización, el empleo del paradigma HABLÉ para referirnos al pasado implica menor grado de subjetividad que el empleo del paradigma HABLO. Usando el 'pretérito indefinido', el control perceptivo del hablante no se desplazará virtualmente y quedará unido con el momento de la enunciación. Si bien el proceso mismo (el cilindro) no los incluye, el control perceptivo y el momento de la enunciación representan el punto de referencia clave para la localización temporal del evento. El uso del paradigma HABLÉ implica que el proceso se sitúa fuera del dominio de control perceptivo del hablante y dentro del dominio de su control epistémico, concretamente en el marco de la realidad conocida. Para poder establecer los límites de la realidad conocida, hemos de tener en cuenta la situación actual del hablante (la base) y orientar el proceso en relación con ella. De esta forma, el conceptualizador resultará más saliente que en el caso del empleo del paradigma HABLO, que no supone este tipo de anclaje, ya que el control perceptivo del hablante desaparece del momento de la enunciación y se coloca virtualmente en el pasado.

\subsection{Posterioridad actualizada}

$\mathrm{Al}$ referirse al futuro, el paradigma HABLO se asocia con procesos planeados que se sitúan en un futuro no muy lejano:

(20) Mañana tengo examen.

Casas, Myrna. Voces. Puerto Rico, 2001, ficción (teatro). 
Matte Bon (2006, 2007) observa que el empleo del paradigma HABLO para referirse al futuro es, además, típico de las situaciones comunicativas en las que se acepta cierta propuesta o se responde a una petición.

Igual que en el caso de la anterioridad actualizada, dichos usos del paradigma HABLO se asocian con procesos cuya extensión temporal no coincide (ni siquiera parcialmente) con el momento del habla. Los dos usos se asimilan a la simultaneidad perceptiva virtual e implican el desplazamiento virtual del dominio de control perceptivo fuera de la propia situación comunicativa a fin de que un proceso situado efectivamente fuera del momento actual pueda ser presentado como controlado por el hablante. No obstante, la diferencia entre la anterioridad y posterioridad actualizadas no consiste solamente en la dirección de dicho desplazamiento (pasado vs. futuro), sino también en el alcance del control perceptivo del hablante, su papel en la representación del proceso y, por consiguiente, en la posible distancia temporal entre el proceso y el momento de habla. Dada la naturaleza del conocimiento humano, el control virtual referido a sucesos ya pasados puede asociarse con procesos muy distanciados no tan solo de la situación comunicativa, sino también de la propia vida del hablante y su experiencia personal. Al hablar del futuro, del cual no podemos tener conocimiento seguro, el control perceptivo virtual está limitado por las previsiones basadas en la experiencia. El alcance temporal de dicho control sería, por tanto, limitado, haciendo que la anterioridad y la posterioridad actualizadas no puedan concebirse como análogas:

En 1492 Colón \{descubrió descubre\} América.

En 2500 el hombre \{descubrirá * descubre\} la vida en otros planetas. 4

Este hecho se refleja también en la representación gráfica en la que el control virtual del hablante (representado por el cubo de rayas intermitentes) tiene que encontrarse en la cercanía del momento de habla:

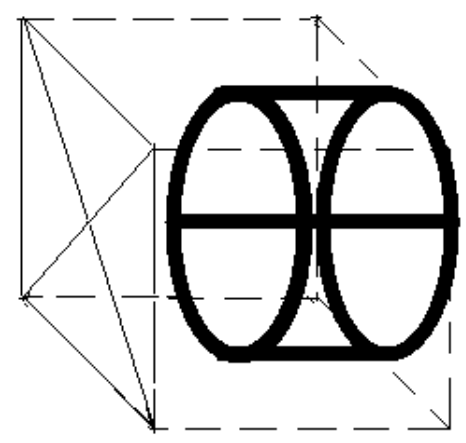

Figura 11. Posterioridad actualizada

El cilindro que representa el proceso verbal no comprende el plano del momento de la enunciación. No obstante, dicho plano corresponde a una de las caras del cubo. Así se ilustra el hecho observado por Matte Bon de que el empleo del paradigma HABLO para expresar procesos futuros se relaciona típicamente con situaciones en las cuales el proceso en cuestión tiene alguna conexión con la situación comunicativa precedente (o, en otras palabras, está vinculado de alguna manera con el momento de la enunciación) y la aserción de la RAE (2009, 
1720), que asocia el llamado 'presente prospectivo' con “afirmaciones rotundas, la descripción de planes, actuaciones previstas o programadas y otros sucesos venideros cuyo acaecimiento no se pone en duda" (o sea, con procesos que forman parte de las previsiones que tiene el hablante en el momento de la enunciación).

Los límites del cilindro se corresponden con los límites del cubo del control perceptivo virtual. Dado que el cilindro no puede sobrepasar los límites de dicho control, su extensión temporal también se ve reducida:

$\{$ Dentro de unas horas $\sim$ Mañana $\sim$ Dentro de un mes $\sim$ ??Dentro de un año $\sim$ ??Dentro de diez años\} estoy en Madrid.

El hecho de que la posterioridad actualizada se vincule con procesos cuya realización se basa en la experiencia o en las presuposiciones del hablante (muchas veces también en la situación comunicativa precedente), y que su ubicación temporal no se pueda alejar demasiado de la situación comunicativa en la que efectivamente se encuentra el conceptualizador, hace que este se perciba más objetivamente y se incorpore más en el proceso que en el caso de la anterioridad actualizada. El grado de subjetivización, si bien es más alto que con los usos del paradigma HABLO que expresan algún tipo de simultaneidad, es por lo tanto menor que en el caso de la anterioridad actualizada.

\subsection{Uso deóntico del paradigma HABLO}

Tradicionalmente se le ha llamado 'presente de mandato' (RAE, 2009, 1720) al empleo deóntico del paradigma HABLO. Dicho uso es posible tanto para expresar una orden o un mandato fuertes como para dar instrucciones o presentar sugerencias o peticiones:

(21) Llamas a esa chica, que más que muerta estará hasta el gorro de aguantar tus misivas, y te disculpas por tantas intromisiones y amenazas. Le pides perdón, y si es verdad que lograste que enfermara, no sólo se lo pides a ella, se lo requieres también a sus padres. Díez, Luis Mateo. Los frutos de la niebla. España, 2008, ficción (relato).

(22) Sólo das vuelta por la Rosales, pasas el auditorio, giras a la derecha y verás la esquina de mi casa; no te puedes perder.

Barrera, Josué. Pasajeros. México, 2010, ficción (relato).

Para poder representar este uso gráficamente, primero es necesario precisar las diferencias entre el imperativo en sí (iPídele perdón!), el uso deóntico del paradigma HABLARÉ (iLe pedirás perdón!) y el empleo del paradigma HABLO para expresar la imposición de una voluntad (iLe pides perdón!). Comparando los tres enunciados, observamos que todos implican el control efectivo del hablante sobre el proceso en cuestión. La diferencia entre ellos consiste en la relación entre el proceso y los dominios de control perceptivo y control epistémico. Las figuras 12, 13 y 14 reflejan la diferencia semántica entre dichas formas de mandato. Como siempre, el cubo representa al hablante en la situación comunicativa y su participación perceptiva en el proceso, mientras que el cilindro corresponde al proceso verbalmente representado. El control efectivo lo representan las flechas. 


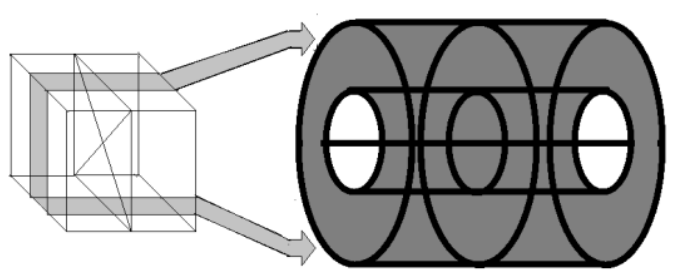

Figura 12. iPídele perdón!

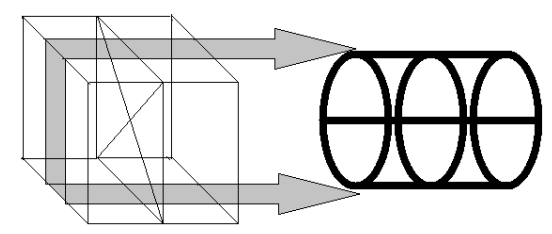

Figura 13. iLe pedirás perdón!

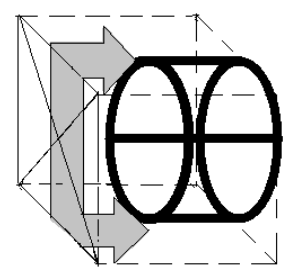

Figura 14. iLe pides perdón!

Como constatamos en el apartado 3, las formas del imperativo (el paradigma iHABLA!) se encuentran fuera del dominio de control epistémico del hablante, ya que se refieren a procesos que no se pueden dar por seguros en el momento de la enunciación y no forman parte de la concepción de la realidad que tiene el hablante. Por el contrario, los paradigmas HABLO y HABLARÉ se refieren a procesos que entran en el dominio de control epistémico.

La esfera de control epistémico viene representada por la extensión vertical del cilindro. Los cilindros más pequeños cuya extensión vertical se corresponde con la del cubo representan los procesos que están sometidos al control epistémico del hablante. De esta forma queda de manifiesto que todos los aspectos del proceso pueden ser observados o procesados por parte del hablante y ser, por tanto, aceptados como reales. Debido a que, al formular un mandato, el hablante puede causar la creación de un proceso que en el momento de la enunciación todavía no puede concebirse como real (no forma parte del dominio de control epistémico), el dominio de control efectivo tiene que ser más extenso que el dominio de control epistémico. La extensión de dicho dominio viene representada por la extensión de las flechas que pueden sobrepasar los límites del cubo.

Volviendo a las representaciones gráficas, observamos que en el caso de iPídele perdón! (figura 12) el cilindro sobrepasa los límites del cubo, pero no sobrepasa los límites del control efectivo representado por las flechas. En el marco del dominio de control epistémico se encuentra solo una pequeña parte del proceso (la que corresponde a las expectativas acerca del futuro y las suposiciones del hablante basadas en su experiencia). Esta parte del cilindro está de nuevo 'iluminada' por la experiencia del conceptualizador, mientras que las partes del 
proceso que sobrepasan el control epistémico del hablante se encuentran 'a oscuras' y corresponden a las partes del proceso que no pueden ser previstas (incluido el cumplimiento o no de la orden, o sea, la realización misma del proceso).

$\mathrm{Al}$ formular una orden con el paradigma HABLARÉ (figura 13), la extensión vertical del cilindro se reduce, ya que el hablante presenta el proceso como si fuese cierto y ya formase parte de su realidad proyectada (de ahí la fuerte imposición que implica el uso deóntico de este paradigma). Si se tratara de una aserción (Mañana, Mario le pedirá disculpas a Luisa), en la representación gráfica faltarían las flechas del control efectivo.

Finalmente, al emplear el paradigma HABLO (figura 14), el hablante no representa el proceso tan solo como cierto, sino también como un proceso en el que se involucrará, por lo menos virtualmente. iMañana le pides perdón! difiere de iMañana le pedirás perdón! en la noción del control perceptivo virtual que expresa el paradigma HABLO. La participación perceptiva virtual del hablante se representa con el cubo de rayas intermitentes. Igual que en el caso de la posterioridad actualizada, la presencia virtual del hablante también se traduce en una reducida distancia temporal entre el momento de habla y el momento en el futuro cuando efectivamente se realiza el proceso. El paradigma HABLARÉ, que no implica la presencia del hablante, puede referirse en su uso deóntico a futuros muy lejanos. El uso del paradigma HABLO sería poco natural en los mismos contextos:

iMañana le \{pedirás pides\} perdón!

iLe \{pedirás ??pides\} perdón todos los días de tu vida!

iNo \{matarás ??matas\}!

6.9. El paradigma HABLO en las oraciones condicionales

El empleo del paradigma HABLO en las pautas como

(23) Si no viene, la encontraremos en el departamento.

Palacios, Óscar. El color de la cebra. México, 2004, ficción (novela).

(24) Tú y él van a ser más felices si vive con nosotros.

Rodríguez, Gustavo. La risa de tu madre. Perú, 2003, ficción (novela).

es un tema muy tratado en la literatura lingüística. En el marco de la pragmática y aplicando el concepto de dominio, Mejías-Bikandi (2009) estudia la imposibilidad de sustituirlo por el paradigma HABLE, que a su vez se emplea con otras conjunciones condicionales como siempre que, como o con tal de que. Su análisis aporta conclusiones convincentes acerca de la conjunción si, que según el autor no funcionaría propiamente como una conjunción condicional, sino más bien como una partícula que crea un dominio paralelo similar al concepto de espacios mentales (mental spaces) presentado previamente por Fauconnier $(1985 ; 1997)$.

Si bien dicho empleo del concepto de dominio explica la selección modal en las oraciones condicionales, no responde explícitamente a la pregunta de por qué el paradigma HABLO sería insustituible por el paradigma HABLARÉ. Analizando este fenómeno, Veiga (1991, 233-234) observa que en las oraciones condicionales con si

[1] os contenidos de las funciones temporales /presente/ y /futuro/ [...] pasan ambos a ser expresados por la forma canto. Al no ser ya funcional la oposición, concluimos que la inflexión de 
si provoca una neutralización contextual de la oposición temporal /presente/-/futuro/, confirmando el carácter no marcado de /presente/ frente a /futuro/ y la formulación de \pm posterioridad como la correcta para dicha oposición de acuerdo con su marca.

Siguiendo la línea de investigación de Mejías-Bikandi y retomando el argumento que defendemos en este artículo, sostenemos que el empleo del paradigma HABLO en vez del paradigma HABLARÉ corresponde a la representación virtual del dominio de control perceptivo, rechazando de este modo la explicación basada en su supuesto carácter no marcado.

Tal y como hemos visto en las representaciones gráficas, el dominio de control perceptivo del hablante no tiene que estar siempre vinculado al momento de la enunciación y puede trasladarse virtualmente tanto al futuro como al pasado, reduciendo de este modo al mínimo la presencia objetiva de la base en la estructuración conceptual y elevando el grado de subjetivización. Dicha dislocación del dominio de control perceptivo no se tiene que realizar solo en el marco de la línea temporal real. El hablante puede ejercer su control virtual también sobre procesos posibles situados en la línea temporal paralela que describe Mejías-Bikandi:

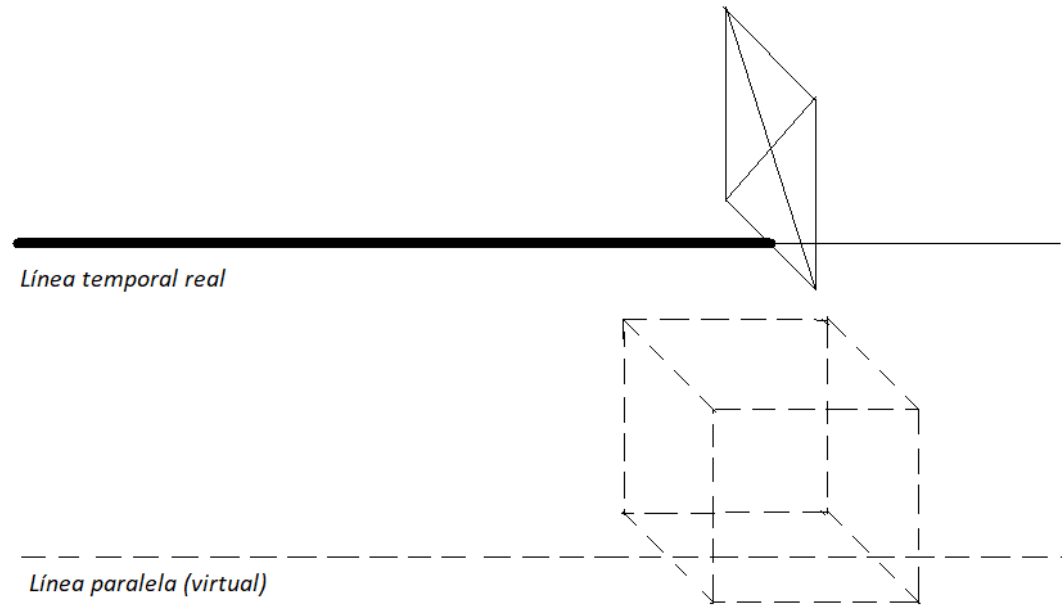

Figura 15. Desplazamiento del control perceptivo hacia la línea temporal paralela

Como hemos indicado, el valor central del paradigma HABLO sería el de señalar la coincidencia (total o parcial) de la extensión temporal de un proceso con el dominio de control perceptivo del hablante (real o virtual). Los paradigmas HABLÉ y HABLARÉ se encuentran fuera de este dominio, donde el paradigma HABLÉ se relaciona con procesos situados en el marco de la realidad conocida (a la izquierda del plano que señala el momento de la enunciación) y HABLARÉ, en su uso temporal, se relaciona con procesos situados en la realidad proyectada (a la derecha del momento de la enunciación, en nuestra representación). Si bien el control perceptivo puede trasladarse virtualmente, el momento de la enunciación es inamovible porque marca el instante real en que se pronuncian ciertas palabras, funcionando así como el punto de anclaje central. Dado que no se encuentra en la línea paralela creada por si, el cubo del control perceptivo no estará anclado a ningún punto de esta línea, por lo que desaparece también la oposición entre pasado y futuro. La tricotomía original HABLÉ HABLO - HABLARÉ se verá reducida a dos elementos: HABLÉ (procesos que no se encuentran bajo el control perceptivo) y HABLO (procesos que entran total o parcialmente en dicho 
dominio). La extensión del cilindro sería variable, dependiendo de la naturaleza del proceso verbal, pero por lo menos una parte correspondería con la extensión del cubo:

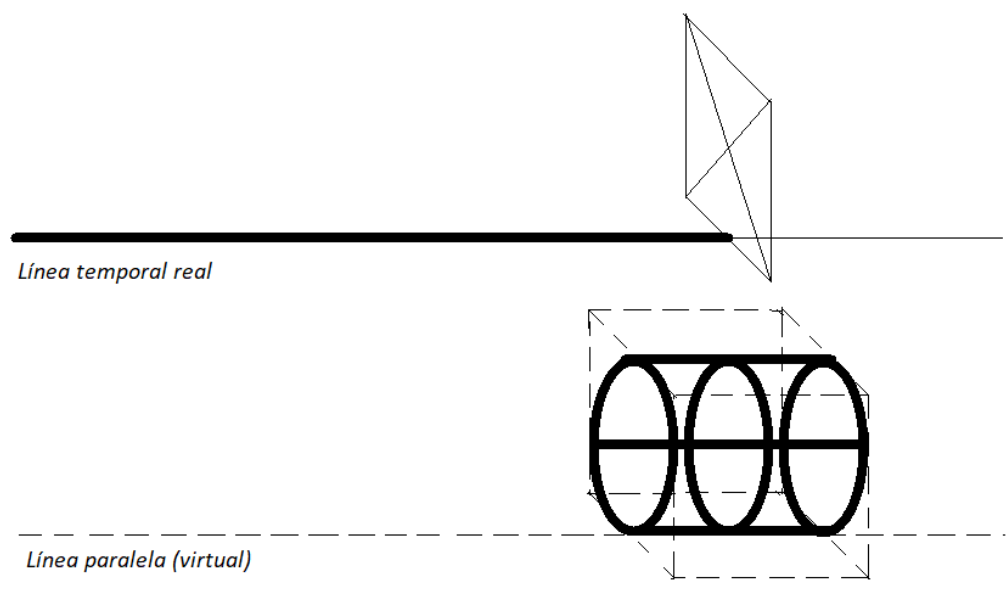

Figura 16. Si no viene, la encontraremos en el departamento.

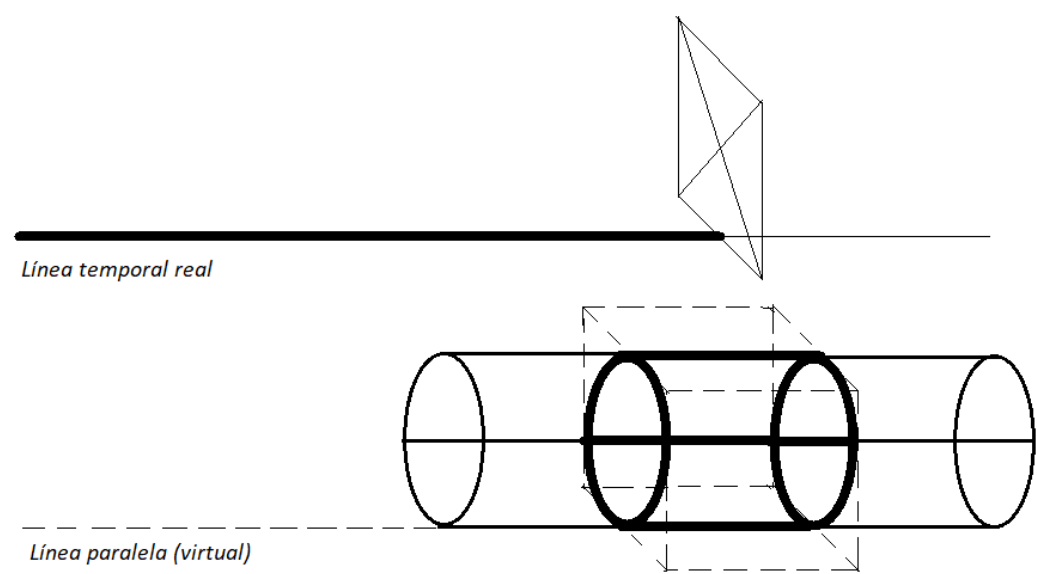

Figura 17. Tú y él van a ser más felices si vive con nosotros.

Dado que el plano del momento de la enunciación no entra en relación con el proceso y se encuentra completamente fuera de la línea en la que este se situaría, se construye con la mayor subjetividad posible.

\section{CONCLUSIONES}

En los apartados anteriores analizamos los distintos usos del paradigma HABLO centrándonos en lo que consideramos su valor central: la coincidencia (total o parcial) del proceso con el dominio de control perceptivo del hablante (real o virtual). Llegamos a la conclusión de que dicha coincidencia se da con todos los usos de este paradigma, tanto con los que implican algún grado de simultaneidad real con el momento de la enunciación, como con los usos considerados tradicionalmente como dislocados, donde el alcance del control perceptivo se corresponde con la diferencia semántica entre el uso del paradigma HABLO y las demás formas verbales. Aplicando el concepto de dominio de control perceptivo a las oraciones 
condicionales, se explica también el hecho de que el paradigma HABLO no sea sustituible por HABLARÉ, sin necesidad de recurrir a explicaciones basadas en el carácter no marcado del presente.

Los análisis han demostrado que el grado de inclusión de la base y del punto de perspectiva del hablante está estrechamente unido al dominio de control perceptivo. Dicho concepto permite, además, concebir tridimensionalmente toda la situación comunicativa en la que se encuentra el hablante y separarla claramente del momento de la enunciación. Esta separación abre la posibilidad de analizar con más detalle el papel del hablante en cada uno de los usos del paradigma HABLO. Asimismo, hemos intentado aportar una nueva perspectiva para el estudio de la subjetivización al trabajar con dos anclajes o dos bases: el anclaje al momento de enunciación real y el anclaje virtual representado por el control perceptivo virtual. En líneas muy generales, podemos constatar que cuanto más unidas se encuentren estas dos bases, menor será el grado de subjetivización y más saliente resultará la perspectiva del hablante en la estructuración conceptual. El análisis de los distintos usos del paradigma HABLO en relación con el grado de subjetivización demuestra, asimismo, la importancia de este concepto y las posibilidades de emplearlo como criterio clave de una nueva clasificación de los modos y tiempos verbales y sus respectivas funciones.

En el caso del paradigma HABLO, el grado de subjetivización iría aumentando con arreglo a la siguiente escala:

Simultaneidad absoluta $\rightarrow$ simultaneidad perceptiva $\rightarrow$ simultaneidad perceptiva virtual $\rightarrow$ simultaneidad extensiva $\rightarrow$ simultaneidad atemporal $\rightarrow$ uso deóntico del paradigma HABLO $\rightarrow$ posterioridad actualizada $\rightarrow$ anterioridad actualizada $\rightarrow$ uso del paradigma HABLO en las oraciones condicionales. 5

Esta escala mantiene la tradicional dicotomía entre usos centrales y usos dislocados del paradigma HABLO. Sostenemos que dicha oposición consiste en la coincidencia o no del momento de la enunciación con el dominio de control perceptivo. En el marco de los usos donde se da dicha coincidencia, el grado de subjetivización se refleja en la extensión temporal del proceso en cuestión (los procesos que suponen mayor extensión temporal implican, asimismo, mayor grado de subjetivización y menor saliencia de la base). En el marco de los usos dislocados, el grado de subjetivización se relaciona con la distancia entre el momento de la enunciación y el dominio de control perceptivo: cuanto más independientes y alejados se encuentren, mayor será el grado de subjetivización. El grado máximo de subjetivización lo representan las oraciones condicionales donde el momento de la enunciación se encuentra fuera de la línea temporal en la que se sitúa el control perceptivo virtual del hablante, haciendo que el momento de la enunciación (y, por tanto, también el anclaje temporal) resulten completamente implícitos y no salientes en la estructuración conceptual.

\section{NOTAS}

1 Para la aplicación de dicho concepto al español, cf. Achard (2000) o Cornillie (2006).

2 Somos conscientes de que el momento de la enunciación también cuenta con cierta extensión temporal. No obstante, tal extensión suele contarse en unos pocos segundos (o incluso menos) y, 
por lo tanto, es lo más cercana posible a la noción de actualidad, en contraposición con el paso del tiempo y su procesamiento por parte del conceptualizador.

3 Para un análisis de esta problemática desde la perspectiva de las funciones comunicativas posibilitadas por los valores gramaticales de HABLO y ESTOY HABLANDO, cf. Laurencio Tacoronte (2016).

4 La pauta En 2500 el hombre descubre la vida en otros planetas solo sería aceptable en un libro de ciencia ficción en que la trama se desarrolle, por ejemplo, en el año 3000 y se trataría de anterioridad actualizada con respecto al año 3000 en el que se sitúa el narrador.

5 Un análisis contrastivo de los usos del 'presente' en distintos idiomas rebasa las expectativas de este artículo. Sin embargo, sostenemos que podría aportar interesantes atribuciones al estudio de la organización de la categoría 'subjetividad' incluso en el sistema verbal de idiomas emparentados. Por ejemplo, la posibilidad de emplear las formas del 'futuro de indicativo' en las prótasis condicionales en italiano y en rumano muestra, a la luz de las observaciones presentadas, una diferencia en la concepción de los elementos de la base y en las posibilidades de su desvinculación.

\section{REFERENCIAS BIBLIOGRÁFICAS}

ACHARD, M. 2000. "Selección de modo en construcciones oracionales de complemento", Revista Española de Lingüística Aplicada, 1, pp. 153-173.

BERMÚDEZ, F. 2006. Evidencialidad. La codificación lingüística del punto de vista. Tesis doctoral. Estocolmo: Stockholms Universitet.

BERMÚDEZ, F. 2011. "El pluscuamperfecto como marcador evidencial en castellano", en Hernández Socas, E., Sinner, C., y Wotjak, G. (eds.), Estudios de tiempo y espacio en la gramática española. Frankfurt am Main: Peter Lang, pp. 43-62.

BERMÚDEZ, F. 2016. "Rumores y otros malos hábitos. El condicional evidencial en español", Cuadernos de Lingüística de El Colegio de México, 3(2), pp. 35-69.

BRIZ, A. 2004. "Notas sobre los llamados usos temporales 'dislocados' en la conversación coloquial”, ELUA: Estudios de Lingüística. Universidad de Alicante, Anexo 2, pp. 43-53.

CASTAÑEDA CASTRO, A. 2004. "Una visión cognitiva del sistema temporal y modal del verbo en español”, ELUA: Estudios de Lingüística. Universidad de Alicante, Anexo 2, pp. 55-71.

CORNILLIE, B. 2006. "Conceptual and constructional considerations on the subjectivity of English and Spanish modals”, en Athanasiadou, A., Canakis, C., y Cornillie, B. (eds.), Subjectification: various paths to subjectivity. Berlín/Boston: Mouton de Gruyter, pp. 177-205.

FAUCONNIER, G. 1985. Mental spaces. Cambridge: MIT Press.

FAUCONNIER, G. 1997. Mappings in thoughts and language. Nueva York: Cambridge University Press.

KRATOCHVÍLOVÁ, D. 2013. "Las relaciones entre el significado modal real y el significado modal potencial en español”, Linguistica Pragensia, 19(2), pp. 73-83.

KRATOCHVÍlOVÁ, D. 2018 (en prensa). Modality in Spanish and combinations of modal meanings. Praga: Karolinum.

LANGACKER, R. W. 1990. Concept, image, and symbol: the cognitive basis of grammar (2). Berlín/Boston: Mouton De Gruyter.

LANGACKER, R. W. 1991. Foundations of cognitive grammar. Volume II: Descriptive application. Stanford: Stanford University Press.

LANGACKER, R. W. 1999. Grammar and conceptualization. Berlín/Boston: Mouton De Gruyter. 
LANGACKER, R. W. 2006. "Subjectification, grammaticization, and conceptual archetypes", en Athanasiadou, A., Canakis, C., y Cornillie, B. (eds.), Subjectification: various paths to subjectivity. Berlín/Boston: Mouton De Gruyter, pp. 17-40.

LANGACKER, R. W. 2009. Investigations in cognitive grammar. Berlín: Mouton de Gruyter.

LANGACKER, R. W. 2017. "Evidentiality in cognitive grammar", en Marín Arrese, J. I., Haßler, G., y Carretero, M. (eds.), Evidentiality revisited. Amsterdam/Filadelfia: John Benjamins, pp. 13-55.

LAURENCIO TACORONTE, A. 2016. “¿Designan las formas gramaticales algo más allá de la misma lengua? El caso del presente de indicativo y el presente de la perífrasis \{estar + -ndo\} en español", Acta Universitatis Carolinae. Philologica, 3, pp. 183-195.

MATTE BON, F. 2006. "Maneras de hablar del futuro en español entre gramática y pragmática. Futuro, ir + infinitivo y presente de indicativo: análisis, usos y valor profundo", RedELE, 6 .

MATTE BON, F. 2007. "Las maneras de hablar del futuro en español: del sistema codificado a las interpretaciones contextuales”, MarcoELE: Revista de Didáctica Español Lengua Extranjera, 5. Disponible en: https://marcoele.com/las-maneras-de-hablar-del-futuro-en-espanol-delsistema-codificado-a-las-interpretaciones-contextuales/

MEJÍAS-BIKANDI, E. 2009. "Conditional sentences and mood in Spanish", Journal of Pragmatics, 41(1), pp. 163-172.

RAE. 2016. Corpus del Español del Siglo XXI (CORPES). Disponible en: http://www.rae.es.

RAE. 2009. Nueva gramática de la lengua española. Madrid: Espasa.

RUIZ CAMPILLO, J. P. 2014. "La lógica del espacio. Un mapa operativo del sistema verbal en español", Journal of Spanish Language Teaching, 1(1), pp. 62-85.

VEIGA, A. 1987. "El presente histórico como hecho de sistema verbal", Verba, 14, pp. 169-216.

VEIGA, A. 1991. Condicionales, concesivas y modo verbal en español. Santiago de Compostela: Universidad de Santiago de Compostela.

VESTERINEN, R. 2014. "Extending the dominion of effective control - Its applicability to mood choice in Spanish and Portuguese", Cognitive Linguistics, 25(4), pp. 583-616.

\section{*AGRAdECIMIENTOS}

Este trabajo ha sido financiado por el Fondo Europeo de Desarrollo Regional - Proyecto "Creatividad y adaptabilidad como condiciones del éxito de Europa en un mundo interrelacionado" (CZ.02.1.01/0.0/0.0/16_019/0000734).

\section{NOTA SOBRE LAAUTORA}

Dana Kratochvílová es doctora en Filología Española y profesora en la Facultad de Letras (Filozofická fakulta) de la Universidad Carolina, República Checa. ORCID: 0000-0002-7050-7363 